\title{
Per-survivor Based Detection of DPSK Modulated High Rate Turbo Codes Over Rayleigh Fading Channels
}

\author{
Bin Zhao and Matthew C. Valenti \\ Lane Dept. of Comp. Sci. \& Elect. Eng. \\ West Virginia University \\ Morgantown, WV 26506-6109 \\ email: bzhao@csee.wvu.edu,mvalenti@wvu.edu
}

\begin{abstract}
In this paper we investigate the performance of a new method for implementing high rate turbo codes over complex fading channels with unknown states. The proposed system features the serial concatenation of an outer punctured turbo code and an inner accumulator separated by a nonuniform interleaver. Per-survivor based APP demodulation and turbo processing are applied to achieve iterative channel estimation and decoding under unknown channel states.
\end{abstract}

Keywords- DPSK, Turbo code, Extended turbo DPSK, Per-survivor processing.

\section{INTRODUCTION}

Turbo codes can achieve remarkable performance in AWGN and flat fading channels with perfect channel estimation. However in non-coherent and partiallycoherent channels, a severe penalty in energy efficiency occurs [1], [2]. This is mainly due to the fact that at low Es/No, there is a big gap in energy efficiency between coherent detection and non-coherent detection techniques where most code concatenation schemes operate. In the recent effort to achieve near coherent performance in unknown or time-varying channels where absolute coherent detection is difficult, several major techniques have been proposed. In [3], [4], pilot symbols have been applied in conjunction with coherent detection to estimate the channel. After initial channel estimation using pilot symbols, the decoded data is used along with the pilot symbols to refine the channel estimate iteratively. In [5], the phase of the fading channel is modeled as a finite-state machine or Markov chain. The channel model and the turbo code trellis are combined to form a code concatenation scheme along which the channel and data are jointly estimated.

In [6], a DPSK modulated convolutional code is modeled as a serially concatenated code and termed 'Turbo DPSK'. The proposed "a posteriori probability (APP) DPSK demodulator" incorporates per-survivorprocessing [7] and linear prediction for channel estimation within the MAP algorithm. Simulation curves show the proposed coded DPSK system appears to per-

\footnotetext{
This work was supported by the Office of Naval Research under grant N00014-00-0655.
}

form asymptotically as well as coded coherent PSK in an AWGN channel, while it can marginally outperform (coded) coherent PSK in Rayleigh fading due to the time diversity introduced by the differential encoder.

In [8], we extended the work of [6] by replacing the outer convolutional code with a turbo code and termed the technique "extended turbo DPSK". The extended turbo DPSK technique performs worse than ideal coherently detected turbo codes with BPSK modulation, however the energy gap tends to decrease as the code rate increases in both AWGN and fully interleaved fading channels. The threshold box analysis shows that the energy inefficiency of extended turbo DPSK results from the undesirable performance of APP DPSK modulation at low Es/No, which is where turbo codes typically operate. Increasing the coding rate will diminish the gap between the ideal turbo code and extended turbo DPSK, because higher rate turbo codes work in a relatively higher Es/No region where the energy gap between APP DPSK and coherent BPSK demodulation shrinks.

Based on our previous work, this paper is dedicated to further investigating the performance of extended turbo DPSK at relatively higher code rates in both AWGN and correlated fading channels. Per-survivor based APP demodulation will be applied along with turbo processing to achieve iterative channel estimation and decoding under unknown channel states.

\section{System Model}

In our system model shown in Fig. 1, we introduce an similar coding scheme as [6]. The major difference is that in our model, a $\mathrm{K}=5$ turbo code $(1,23 / 35,23 / 35)$ rather than a convolutional code is applied as the outer code. To investigate the performance of higher rate codes, we introduce an optimal rate compatible puncturing pattern from [9] for the outer turbo code with a framesize of 1024 bits. Both constituent codes are terminated with independent tails. The design of our turbo interleaver follows [10], which guarantees a sense of structural pseudo-randomness.

Conceptually, we consider the DPSK modulator to be a recursive rate 1 convolutional inner code, which in 


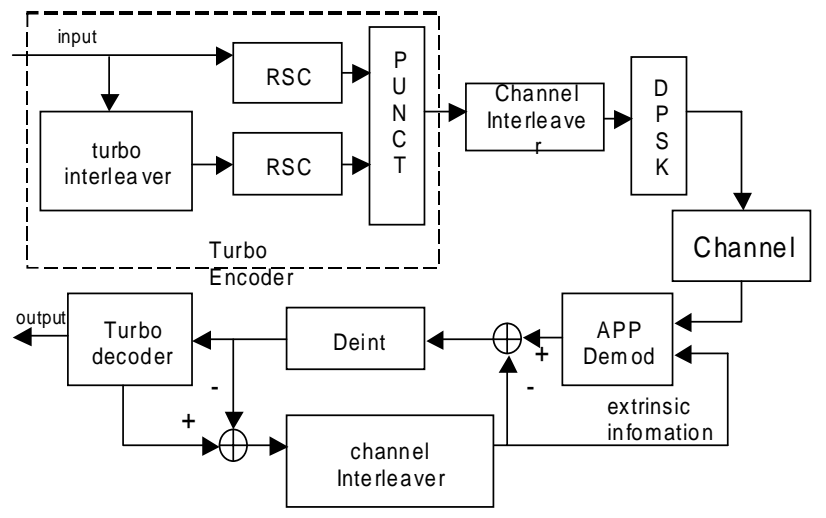

Fig. 1. System model for serial concatenation of turbo code and DPSK modulation.

turn can be represented by a two-state trellis. For unknown channel states, the 'APP DPSK demodulator' is proposed to incorporate per-survivor processing and linear prediction into the MAP algorithm to simultaneously achieve channel estimation and demodulation. A fixed length time window sliding over multiple adjacent stages of the simple DPSK trellis constructs a super-trellis for this 'APP demodulator'. The size of the sliding window equals the order of linear prediction, and the complexity of the demodulator is exponentially proportional to the size of sliding window. For instance, if the order of prediction coefficients is $m$, then the total number of states in the super-trellis is $2^{m}$. Thus the size of the prediction filter must be rather small to be practical (only about 3 or 4 coefficients). With perfect channel estimation, the 'APP DPSK' super-trellis is reduced to a simple DPSK twostate trellis without losing optimality. The availability of soft outputs from this demodulator makes possible the outer MAP decoding and turbo processing according to the serially concatenated model.

The turbo interleaver provides interleaving gain due to its spectral thinning functionality [11], while the channel interleaver will not only offer interleaving gain, but also help to overcome the correlated channel. It is well known that turbo codes will be most effective to correct random errors, while in correlated fading channels, without channel interleaving, the performance is severely degraded. In [2], it is shown that with perfect channel estimation, the fully interleaved channel has at least $3 \mathrm{~dB}$ more energy efficiency at $B E R=10^{-5}$ over that of the correlated Rayleigh fading channel with $\mathrm{BT}=0.01$ and even more over the correlated fading with $\mathrm{BT}=0.001$. If we consider an $\mathrm{M}$ by $\mathrm{N}$ block interleaver, where the data bits are written in along the rows and read out along the columns, then adja- cent errors caused by correlated channel fades will be spread $\mathrm{N}$ bits apart, or equivalently the fading speed of the channel is increased by $\mathrm{N}$ times. Therefore a simple block interleaver helps to significantly improve the performance of turbo codes in correlated fading channels.

The log-MAP [12] algorithm is applied to decode both the inner and outer code. For convenience of explanation, we refer to the iterative decoding just within the turbo decoder as the "local iteration" and the overall iterative DPSK demodulation and turbo decoding as the "overall iteration". The soft output of both inner and outer codes are derived using:

$$
\Lambda_{k}=\log \frac{\operatorname{Pr}\left(X_{k}=1 \mid \mathbf{Y}\right)}{\operatorname{Pr}\left(X_{k}=0 \mid \mathbf{Y}\right)},
$$

where $\Lambda_{k}$ is the log likelihood ratio (LLR) of $X_{k}$, and $\mathbf{Y}$ represents the received channel sequence. $X_{k}$ could be either an input bit or an output bit of the constituent encoder. For the APP DPSK demodulator, $X_{k}$ represents the input information of the DPSK encoder, while for the turbo decoder, $X_{k}$ is the output of the turbo encoder representing either a systematic or a parity bit. (1) can be further broken down into three terms:

$$
\begin{aligned}
\Lambda_{k}= & \log \frac{\operatorname{Pr}\left(Y_{k} \mid X_{k}=1\right)}{\operatorname{Pr}\left(Y_{k} \mid X_{k}=0\right)}+\log \frac{\operatorname{Pr}\left(Y_{i \neq k} \mid X_{k}=1\right)}{\operatorname{Pr}\left(Y_{i \neq k} \mid X_{k}=0\right)} \\
& +\log \frac{\operatorname{Pr}\left(X_{k}=1\right)}{\operatorname{Pr}\left(X_{k}=0\right)} .
\end{aligned}
$$

The third term in (2) is the extrinsic information from the other block in the concatenated scheme. For the DPSK demodulator, it is the extrinsic information from the turbo decoder. To avoid information saturation during iterative processing, it should be subtracted from (2) before being fed into the turbo decoder as a soft input.

The soft output from the turbo decoder has the same form as (1) and (2), however for the turbo code, the LLR value of the extrinsic information is set to zero; therefore the third part in (2) disappears. Within the turbo decoder, only one local iteration is used per overall iteration. The first term in (2) is the soft value passed in from the output of the APP DPSK demodulator. The second term is the extrinsic information generated by the action of the turbo decoder. Therefore, after interleaving, it could be considered to be extrinsic information and fed into the DPSK APP demodulator during the next overall iteration.

A generalized expression for the branch metric used by the APP DPSK demodulator under unknown fading 
channels can be expressed as:

$$
\gamma_{k}\left(\tilde{b_{k}}\right)= \begin{cases}z_{k}-\frac{\left|y_{k}-\tilde{b}_{k} F(\mathbf{Y}, B)\right|^{2}}{2 \sigma^{2}} & \tilde{a}_{k}=1 \\ -\frac{\left|y_{k}-\tilde{b}_{k} F(\mathbf{Y}, B)\right|^{2}}{2 \sigma^{2}} & \tilde{a}_{k}=0,\end{cases}
$$

where $z_{k}$ is the input extrinsic information, $y_{k}$ is the channel output, $\tilde{b}_{k}$ is the estimated channel input (i.e. DPSK encoder's output), $\tilde{a}_{k}$ is the DPSK encoder's input, $\mathrm{F}(\mathbf{Y}, \mathrm{B})$ is the estimated channel gain which is a function of survivor path $\mathrm{B}$ and channel output sequence $\mathbf{Y}$, and $\sigma^{2}$ is the noise variance. Under perfect channel estimation, $\mathrm{F}(\mathbf{Y}, \mathrm{B})$ is the fading coefficient $h_{k}$, while $\sigma^{2}$ is the variance of the Gaussian noise component $\sigma_{n}^{2}$. For an AWGN channel, $F(\mathbf{Y}, B)=1$. As [6] points out, under unknown channel states, $\mathrm{F}(\mathbf{Y}, \mathrm{B})$ is the fading prediction $\tilde{h}_{k}=\sum_{n=1}^{N} p_{n} y_{k-n} \tilde{b}_{k-n}$, where $y_{k}$ is the channel output, $\tilde{b}_{k-n}$ is the hypothesized symbol according to the per-survivor processing, and $p_{n}$ is a Wiener filter coefficient. The noise variance $\sigma^{2}$ includes both Gaussian noise and the prediction error expressed as:

$$
\sigma^{2}=\frac{1}{2}+\sigma_{n}^{2}-\frac{1}{2} \sum_{n=1}^{N} p_{n} r_{n}
$$

where $r_{n}$ is the channel auto-correlation coefficient. As $N \rightarrow \infty, \sigma^{2} \rightarrow \sigma_{n}^{2}$.

\section{Simulation Results}

Simulations were run to illustrate the performance of higher rate extended turbo DPSK under different channel conditions. For the simulation, a framesize of $K=1024$ bits was used. In total, there are five different coding schemes are simulated including BPSK modulated turbo code with interleaver and perfect channel state information (turbo code with interleaver), BPSK modulated turbo code with perfect channel state information (turbo code), DPSK modulated and coherently detected turbo code with interleaver and perfect channel state information (extended turbo DPSK), DPSK modulated turbo code with interleaver and per-survivor based channel estimation (extended turbo DPSK with PSP). DPSK modulated turbo code with interleaver and differential detection (differential turbo DPSK) is also included as a benchmark. For any turbo code with BPSK modulation scheme, up to 15 decoder iterations were performed. For any extended turbo DPSK related schemes, up to 15 global iterations, with 1 local iteration for each global iteration, were performed. Fig. 2 shows BER performance of the turbo code and extended turbo DPSK in AWGN channel, while Fig. 3, Fig. 4 and Fig. 5 shows the BER of extended turbo DPSK at different rates in correlated fading channels.

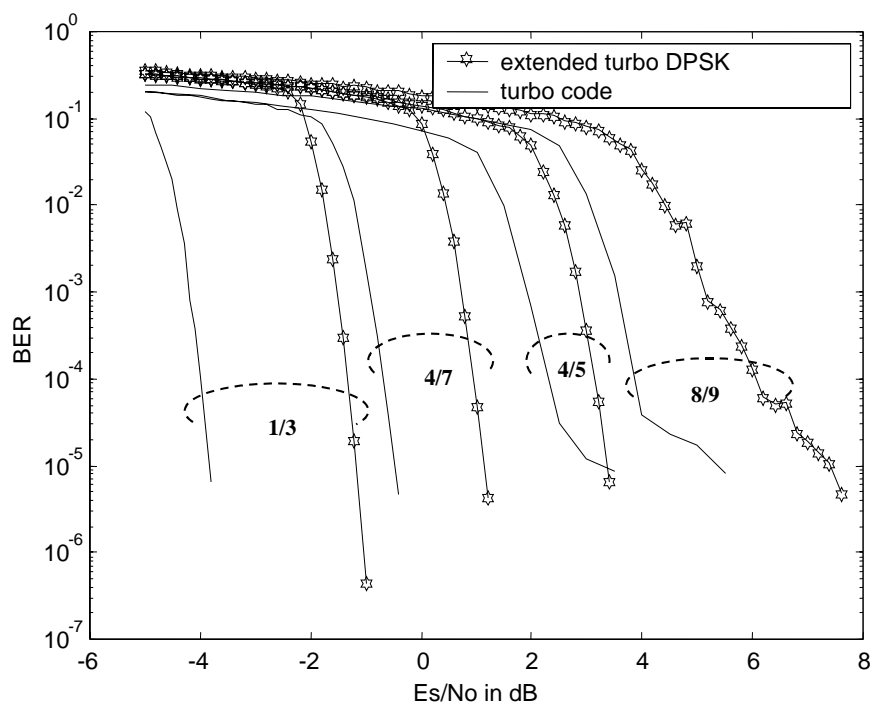

Fig. 2. BER of higher rate codes with the framesize $\mathrm{K}=1024$ in AWGN channel: $1 / 3,4 / 7,4 / 5,8 / 9$.

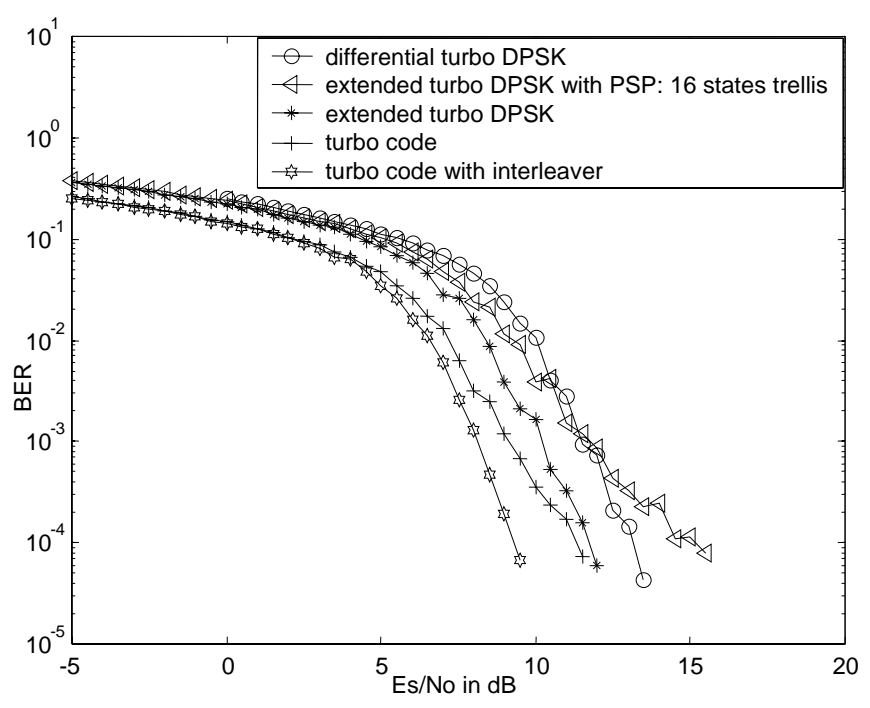

Fig. 3. BER of rate $4 / 5$ codes in fading channel: $\mathrm{BT}=0.01$.

Fig. 2 shows performance consistent with that of [8], although different framesize and code polynomials are applied. The energy gap between turbo code and extended turbo DPSK is about $3 \mathrm{~dB}$ at rate $1 / 3$, while it decreases to about $1 \mathrm{~dB}$ as the rate increases to $4 / 5$. In an AWGN channel, turbo code with interleaver should have the same performance as that of turbo code. One advantage of extended turbo DPSK is that it has a lower error floor, and therefore it performs much better than turbo BPSK at relatively high SNR. However at even higher rate, say rate $8 / 9$, the gap increases again. The performance of all these different code rates can be explained by the convergence box theory [8]. The energy gap between DPSK and BPSK 


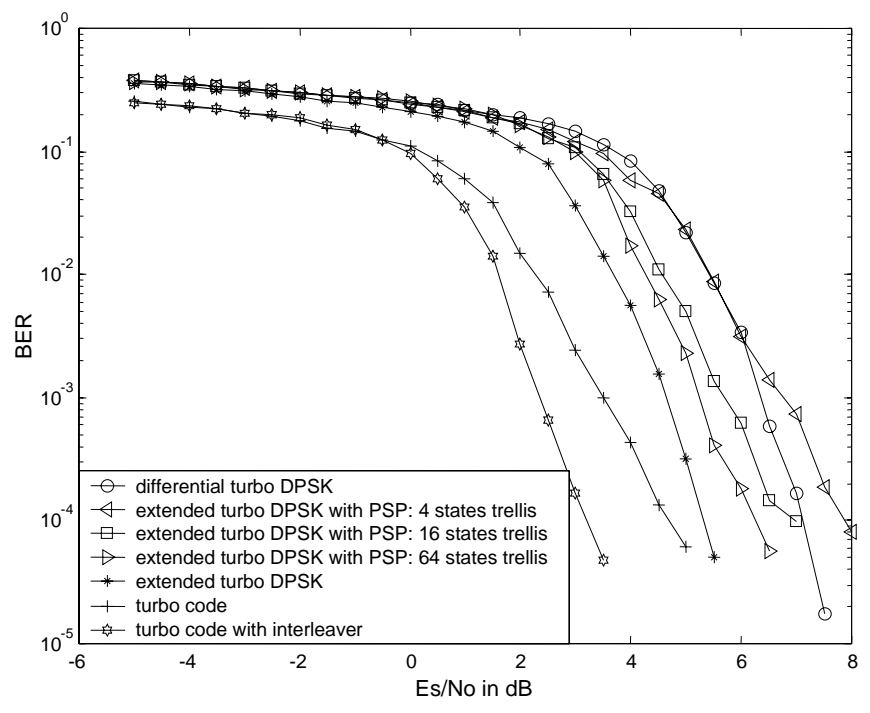

Fig. 4. BER of rate $4 / 7$ codes in fading channel: $B T=0.01$.

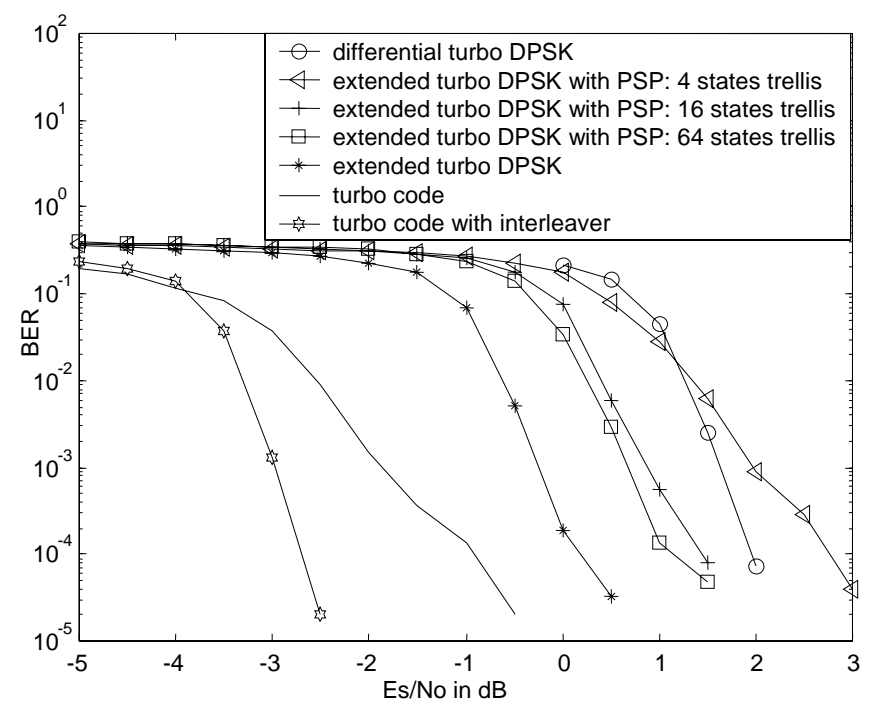

Fig. 5. BER of rate $1 / 3$ codes in fading channel: $\mathrm{BT}=0.01$.

modulation causes extended turbo DPSK to perform generally worse than turbo code at low coding rate. However, the gap between those modulation schemes decrease significantly, therefore the performance curves of turbo code and extended turbo DPSK get closer as the rate gets higher. However the energy gap does not disappear according to our simulation results and actually increases as the rate gets even higher. This phenomenon is probably due to two factors: first of all, the turbo decoding is itself suboptimal; secondly, although a total of 15 global iterations are applied, only 1 local iteration is used within turbo decoding. This decoding scheme might cause the performance to deteriorate as the outer punctured turbo code becomes too weak. In this case, it is better to just use differential detection for extended turbo DPSK at very high rate.

Fig. 3 shows the performance of rate $4 / 5$ codes in correlated fading channel $(\mathrm{BT}=0.01)$. Differential turbo DPSK is about $4 \mathrm{~dB}$ worse than turbo code with interleaver. Block interleaving improves the performance of turbo codes by about $1.5 \mathrm{~dB}$. The extended turbo DPSK scheme loses about $2.5 \mathrm{~dB}$ energy efficiency compared with turbo code with interleaver. It is rather unexpected that extended turbo DPSK with PSP performs $2 \mathrm{~dB}$ worse than differential turbo DPSK at rate $4 / 5$.

Fig. 4 and Fig. 5 show the performance of rate $4 / 7$ and rate $1 / 3$ codes in correlated fading channel $(\mathrm{BT}=0.01)$. The energy gap between turbo code with interleaver and extended turbo DPSK tends to increase as the rate decreases. Differential turbo DPSK is $4 \mathrm{~dB}$ worse than turbo code with interleaver at rate $4 / 7$, and the gap increases to $4.5 \mathrm{~dB}$ at rate $1 / 3$. The block interleaver improves the performance of turbo code by about $1.5 \mathrm{~dB}$. The extended turbo DPSK loses about $3 \mathrm{~dB}$ energy efficiency to turbo code with interleaver at both code rates which becomes the main reason why extended turbo DPSK with PSP is in total, $4 \mathrm{~dB}$ less efficient than turbo code with interleaver. The per-survivor based channel estimation itself only loses about $1 \mathrm{~dB}$ in energy efficiency. This $4 \mathrm{~dB}$ energy gap does not significantly decrease as it does in AWGN channels because in fading channels the energy gap between the BPSK and DPSK modulation is much larger than that of AGWN channel. It could only be partially recovered by the iterative channel estimation and decoding techniques. However, iterative channel estimation and decoding applied in extended turbo DPSK with PSP still offers about $1 \mathrm{~dB}$ processing gain over differential turbo DPSK.

Fig. 4 and Fig. 5 also show the effect of the supertrellis size on the performance of extended turbo DPSK with PSP in the fading channel $(\mathrm{BT}=0.01)$. Increasing the trellis size from 4 states to 16 states does improve the performance by about $1 \mathrm{~dB}$. However, further increasing the trellis size to 64 states provides a decreasing marginal benefit. As pointed out by (4), in APP demodulator, increasing the trellis size is equivalent to increasing the order of linear prediction, which will decrease the prediction error. But the marginal benefit will decrease as the order of linear prediction increases. As the prediction order approaches infinity, the noise variance of APP demodulator will be reduced to Gaussian noise variance. Of course, in this case, the APP demodulator is no longer practical.

The simulation does show some unexpected results. For instance, at very high rate (rate higher than $4 / 5$ ) extended turbo DPSK with PSP performs worse than differential turbo DPSK, and at medium low rate, per- 
survivor processing with a 4-state super-trellis performs worse than differential turbo DPSK. This is probably due to the fact that in the joint estimation and data detection cycle, just 1 local suboptimal turbo decoding is applied which will exasperate the performance of extended turbo DPSK especially when the outer turbo code is quite weak.

\section{CONCLUSiOnS}

In this paper we investigated the performance of turbo codes with DPSK modulation at high rate over complex fading channels with unknown states. In general, the proposed system performs worse than coherently detected BPSK modulated turbo codes with interleaver, as there is an energy gap of about $3 \mathrm{~dB}$ between turbo code with interleaver and extended turbo DPSK. This energy gap decreases as the rate increases in both AWGN and fading channels.

Due to DPSK modulation, the proposed system performs worse than that of pilot symbol based approach of [4]. The energy inefficiency is caused mainly by the following reasons: first of all, there is a big energy gap between coherent detection of DPSK and BPSK modulations at low Es/No. Secondly, just 1 local iteration of the turbo decoder itself is suboptimal, therefore the feedback of suboptimal extrinsic information will further deteriorate the performance especially at higher rate. Finally, although the outer punctured turbo code is optimal in a sense of PCCC, the overall extended turbo DPSK might not be optimal in a sense of serial code concatenation. However, in our investigation we found that per-survivor based detection provides about $1 \mathrm{~dB}$ energy gain over differential turbo DPSK and it only loses $1 \mathrm{~dB}$ to the extended turbo DPSK. We also found that the size of the APP demodulator trellis will affect the performance of channel estimation and code detection in our system.

As for our future research, efforts will be put into the search for optimal puncturing patterns for extended turbo DPSK and a better modulation structure for turbo codes with a convergence region comparable or even better than that of BPSK modulated turbo codes. We also will develop analytical tools that leverage the concepts of Gaussian density evolution [13] and convergence boxes [8] of extended turbo DPSK in the errorcliff region.

\section{REFERENCES}

[1] E. K. Hall and S. G. Wilson, "Turbo codes for noncoherent channels," in Proc., IEEE GLOBECOM, Communication Theory Mini-Conference, (Phoenix, AZ), pp. 66-70, Nov. 1997.

[2] E. K. Hall and S. G. Wilson, "Design and analysis of turbo codes on Rayleigh fading channels," IEEE J. Select. Areas Commun., vol. 16, pp. 160-174, Feb. 1998.
[3] Q. Li, C. N. Georghiades, and X. Wang, "An iterative receiver for turbo-coded pilot-symbol assisted modulation in fading channels," IEEE Commun. Letters, vol. 5, pp. 145147, April 2001.

[4] M. C. Valenti and B. D. Woerner, "Iterative channel estimation and decoding of pilot symbol assisted turbo codes over flat-fading channels," IEEE J. Select. Areas Commun., vol. 9, pp. 1691-1706, Sep. 2001.

[5] C. Komninakis and R. D. Wessel, "Joint iterative channel estimation and decoding in flat correlated Rayleigh fading channels," IEEE J. Select. Areas Commun., vol. 9, pp. 1706-1718, Sep. 2001.

[6] P. Hoeher and J. Lodge, "Turbo DPSK: Iterative differential PSK demodulation and channel decoding," IEEE Trans. Commun., vol. 47, pp. 837-843, June 1999.

[7] R. Raheli, A. Polydoros, and C. K. Tzou, "Per-survivor processing: A general approach to MLSE in uncertain environments," IEEE Trans. Commun., vol. 43, pp. 354-364, Feb./Mar./Apr. 1995.

[8] B. Zhao and M. C. Valenti, "Iterative demodulation and decoding of DPSK modulated turbo codes over Rayleigh fading channel," in 11th Anual Virginia Tech. Symposium on Wireless Personal Communication, pp. 915-921, June 2001.

[9] D. N. Rowitch and L. B. Milstein, "On the performance of hybrid fec-arq systems using rate conpatible punctured turbo (RCPT) codes," IEEE Trans. Commun., vol. 48, pp. 948-959, June 2000.

[10] Eureopean Telecommunications Standards Institute, "Universal mobile telecommunications system (UMTS): Multiplexing and channel coding (FDD)," 3GPP TS 125.212 version 3.4.0, pp. 14-20, Sept. 232000.

[11] S. Benedetto, D. Divsalar, D. Montorsi, and F. Pollara, "Serial concatenation of interleaved codes: Performance analysis, design, and iterative decoding," IEEE Trans. Inform. Theory, vol. 44, pp. 909-926, May 1998.

[12] P. Robertson, P. Hoeher, and E. Villebrun, "Optimal and sub-optimal maximum a posteriori algorithms suitable for turbo decoding," European Trans. on Telecommun., vol. 8, pp. 119-125, Mar./Apr. 1997.

[13] D. Divsalar, "Low complexity turbo like codes," Proc. Second International Symp. on Turbo Codes and Related Topics, pp. 73-80, Sept. 2000.

[14] S. Dolinar and D. Divsalar, "Weight distributions for turbo codes using random and nonrandom permutations," JPL TDA Progress Report, vol. 42, pp. 56-65, Aug. 15th 1995.

[15] R. Ramanurthy and W. E. Ryan, "Convolutional double accumulator codes (or double turbo DPSK)," Electronics Letters, vol. 5, pp. 157-159, Apr. 2001. 